\title{
Primary ovarian insufficiency and fertility preservation: review article
}

\author{
Purohit Prashant ${ }^{1}$, Mike Savvas' ${ }^{1}$, Ibrahim A. Abdelazim², Gulmira Zhurabekova ${ }^{3}$ \\ 'Assisted Reproduction Unit, King's Hewitt Fertility Centre, Kings College Hospital, London \\ 2Department of Obstetrics and Gynecology, Ain Shams University, Cairo, Egypt and Ahmadi Hospital, Kuwait Oil Company (KOC), \\ Ahmadi, Kuwait \\ ${ }^{3}$ Department of Normal and Topographical Anatomy, Marat Ospanov, West Kazakhstan State Medical University (WKSMU), \\ Aktobe, Kazakhstan
}

\begin{abstract}
Introduction: Female fertility and the ovarian reserve decrease with age.

Material and methods: Review of literature about the primary ovarian insufficiency (POI) and methods of fertility preservation to define women at risk of POI and their fertility saving options.

Results: Women with a family history of POI, fragile X syndrome primary ovarian insufficiency mutation (FXPOI), autoimmune thyroiditis, Addison's disease, exposure to toxins and viral infection are at high risk of POI. In-vitro-fertilization success improved after introduction of oocyte (or embryo) vitrification. Transplantation of the cryo-preserved ovarian tissue could be a fertility preservation option. Transplantation of the cryo-preserved ovarian tissue is an outpatient procedure, which restores hormonal function and fertility. After ovarian tissue transplantation women can conceive normally without medical assistance.

Conclusions: The use of donor oocytes is the only treatment for women at risk of POI. The in-vitro-fertilization outcome improved after introduction of oocyte (or embryo) vitrification. Transplantation of the cryo-preserved ovarian tissue is an outpatient procedure, which restores hormonal function and fertility.

Key words: primary, ovarian, insufficiency, fertility, preservation.
\end{abstract}

Corresponding author: Prof. Ibrahim A. Abdelazim, Department of Obstetrics and Gynecology, Ain Shams University, Cairo, Egypt and Ahmadi Kuwait Oil (KOC) Company Hospital, P.O. Box: 9758, 61008 Ahmadi, Kuwait, phone: +965-66551300, e-mail: dr.ibrahimanwar@gmail.com 


\section{Introduction}

Female fertility decreases with age and the female fertile span influenced by the number of the primordial follicles containing diplotene oocytes surrounded by cuboidal granulosa cells arrested in the first meiotic prophase [1]. The reserve of the primordial follicle decreases with age, resulting in cessation of menses and menopause [2]. Premature menopause or premature ovarian failure (POF) - defined as the complete loss of ovarian function occurring before the age of 40 and affecting $1 \%$ of the population [3].

Premature ovarian failure - considered - one of the crucial factors contributing to infertility. The POF occurs due to progressive decrease in the number and the quality of the oocytes sequestered in the primordial follicles [4, 5]. Primary ovarian insufficiency is the phase when there is some loss of ovarian function and may be considered as the initial phase of the POF. During the primary ovarian insufficiency (POI) phase, women may experience regular menses and may able to conceive, but the ovarian function and the fertility reduced. The POI is often diagnosed by the fertility specialist and the presenting woman is usually complaining of sub-fertility or infertility [6].

\section{Causes of primary ovarian insufficiency}

Women at risk of POI: It is estimated that $5 \%$ of women will experience the menopause or ovarian failure by the age of 45 years and researchers estimate that POI affects 1 in 1,000 women by the age of 30 years, 1 in 250 women by the age of 35 years and 1 in 100 women by the age of 40 years [7].

Causes of POI: The exact cause of POI is unknown in the majority of cases and several factors are responsible for the ovarian follicles' depletion or dysfunction or both. $10-20 \%$ of women with POI have a positive family history of POI $[7,8]$. Studies suggest that some genetic mutations are responsible for $28 \%$ of the POI as fragile X syndrome mutation or the mosaic variant of Turner syndrome [7, 9]. Fragile X syndrome mutation at higher risk for POI is called fragile $\mathrm{X}$ syndrome primary ovarian insufficiency mutation (FXPOI) [9]. Autoimmune diseases were suggested as a cause of POI, because $20 \%$ of women with POI have an autoimmune thyroiditis and Addison's disease $[3,10]$. Metabolic disorders were also suggested as a cause of POI, because more than $80 \%$ of women with galactosemia have POI [11]. Toxins, viral infection, cigarette smoking, chemicals and pesticides can speed the ovarian follicle depletion through stimulation of the autoimmune destruction of the ovarian follicles $[12,13]$. The POI may develop after a hysterectomy in spite of ovarian preservation due to disturbed ovarian blood supply after the hysterectomy [14].

\section{Clinical presentation of primary ovarian insufficiency}

Women with POI may present with oligo- or amenorrhea with menopausal symptoms such as hot flushes, night sweats, irritability, insomnia, depression, decreased libido, dyspareunia and vaginal dryness [15]. Osteoporosis is one of the long-term consequences of POI [16] and 54\% of women diagnosed with POI report loss of fertility as the most distressing consequence of their diagnosis [17]. The POI is often diagnosed by the fertility specialist, when the investigations of infertile woman showed elevated follicle stimulating hormone (FSH), low estradiol $\left(\mathrm{E}_{2}\right)$ and low anti-Mullerian hormone (AMH). Many cases of POI presented with unexplained infertility.

\section{Diagnosis of primary ovarian insufficiency}

The key signs for diagnosis of POI are: 1) missed or irregular periods for 4 months after exclusion of unexpected pregnancy; 2) high FSH with low $\mathrm{E}_{2}[10,18]$.

During assessment of women with POI, the physician should confirm or exclude a family history of POI and/or fragile $\mathrm{X}$ syndrome mutation, past history of ovarian, pelvic surgery, pelvic chemo-radiation and the associated endocrine disorders such as Addison's disease or hypothyroidism.

\section{Possible treatment options for primary ovarian insufficiency}

Currently, there is no treatment to restore the normal ovarian function, but the health risks and conditions associated with POI can be reduced. Hormone replacement therapy (HRT) improves the sexual health and decreases the risks of osteoporosis in women with POI [19]. The HRT for women with POI reduces hot flashes and night sweats and maintains bone health [17]. The HRT for women with POI will not prevent pregnancy, and evidence suggests that HRT might improve pregnancy rates by lowering high luteinizing hormone (LH) [20]. The HRT taken by women with POI is different from the HRT taken by women who are going through natural menopause, which is called post-menopausal hormone therapy (PMHT). Unlike PMHT, HRT for POI is not associated with increased risk of strokes, heart diseases and breast cancers [3]. Women with POI are at high risk for osteoporosis and they should get at least 1200-15000 mg of elemental calcium and 1000 IU of vitamin D daily [20]. Maintaining a healthy body weight and regular physical activity are also important factors for reducing the risk of osteoporosis and heart diseases in women with POI [18].

\section{Pregnancy after primary ovarian insufficiency}

Five to $10 \%$ of women with POI get pregnant without medical intervention after the diagnosis of POI. Researchers suggest that "spontaneous remission" may occur after the diagnosis of POI with subsequent return of fertility and pregnancy [21]. There is no effective intervention to enhance the spontaneous or natural conception after POI. Researchers also suggest that $24 \%$ of women with POI will experience intermittent ovarian function and $4 \%$ will conceive spontaneously. Younger women with POI are more likely to conceive spontaneously and women who present with POI and primary amenorrhea are less likely to conceive compared to those who present with secondary amenorrhea [22]. 


\section{Prediction of primary ovarian insufficiency}

There is no reliable way to identify women who will develop POI. Thorough history taking can sometimes be helpful, particularly if the mother or any of the sisters had an early menopause. A recent study from Denmark revealed that women whose parents had an early menopause were more likely to have reduced AMH and antral follicle count (AFC) [23]. A history of autoimmune disease, particularly Addison's and autoimmune thyroiditis, may also increase the likelihood of POI.

Assessment of ovarian reserve can identify women at risk of POI. Ovarian reserve means the reproductive potential of the women through identification of the number and the quality of oocytes in their ovaries.

Decreased ovarian reserve (DOR) describes women of reproductive age whose response to ovarian stimulation or fecundity reduced compared to other women of the same age. It is possible to assess the ovarian reserve with day-3 $\mathrm{FSH}$ and $\mathrm{E}_{2}$ assay, $\mathrm{AFC}$ and $\mathrm{AMH}$.

High day-3 FSH and low $\mathrm{E}_{2}$ suggest DOR. Although the $\mathrm{AFC}$ is operator dependant, it is one of the markers of the ovarian reserve. Serum AMH appears to be more reliable than FSH and AFC. The AMH is gonadotropins independent and its assay can be done at any time of the menstrual cycle. The AMH levels below $1 \mathrm{ng} / \mathrm{ml}$ are associated with DOR [24].

Freeman et al. suggested that the rate of decline in the AMH rather than the absolute AMH level is useful in predicting the age of the menopause [25]. Nikolaou et al. reported that women who had a poor response to ovarian stimulation were likely to develop menopausal symptoms within seven years [26]. Lawson et al. reported that a poor ovarian response was a strong predictor for ovarian failure than elevated FSH [27].

\section{Fertility saving options for women at risk of primary ovarian insufficiency}

The use of donor oocytes is the only treatment option for women with POI with a good pregnancy outcome. A report on artificial reproductive techniques (ART) from ESHRE in 2009 showed that 21604 women in ART programs treated with donor oocytes (double the women treated with donor oocytes in 2005) and the mean age of oocyte recipients was 40 years $[28,29]$.

The scarcity of the donors is the limitation of this form of treatment. Furthermore, this form of treatment is not acceptable to some women and is illegal in some countries. A further aspect of the treatment is oocyte (or embryo) cryopreservation, which is widely used for women prior to cancer treatment [21].

Embryo cryopreservation has decreased the number of fresh embryo transfers and maximized the effectiveness of the IVF cycle. Similarly, embryo cryopreservation is a crucial tool in cases of cancelled embryo transfer (ET) due to ovarian hyperstimulation risk, endometrial bleeding and elevated serum progesterone levels on the day of triggering. Embryo cryopreservation has enhanced the clinical benefits and cumulative conception rates possible for couples following a single cycle of ovarian stimulation and IVF [30].

However, oocyte cryopreservation offers more advantages compared to embryo freezing: 1) fertility preservation in women at risk of losing fertility due to oncological treatment, premature ovarian failure or chronic disease; 2) it can help alleviate religious and/or other ethical, legal and moral concerns of embryo storage; 3) it makes "egg banks and/or egg donations" possible by eliminating donor-recipient synchronization problems; and 4) it allows women to postpone childbirth until a later time/age (after establishing a career) [30].

Vitrification (glass-like state) is an alternative approach to embryo/oocyte cryopreservation which has been recently described as a revolutionary technique; however, the first successful embryo vitrification was published in the middle of the 1980s [31]. Vitrification is different from slow freezing in that it avoids the formation of ice crystals in the intracellular and extracellular space [31]. Vitrification is the solidification of a solution by an extreme elevation in viscosity at low temperatures without ice crystal formation, a process achieved by a combination of a high concentration of cryoprotective additives (4-8 $\mathrm{mol} / \mathrm{l}$ ) and an extremely high (ultra-rapid) cooling rate [32].

In contrast to slow freezing, during vitrification, cells are dehydrated mainly before the start of the ultra-rapid cooling by exposure to high concentrations of cryoprotective additives, which is necessary to obtain a vitrified intracellular and extracellular state afterwards. The potential risk associated with the vitrification procedure is the high concentration of cryoprotective additives that could be toxic to cells. However, it is possible to limit toxicity of cryoprotective additives by mixing different cryoprotective additives, thereby decreasing the relative concentration of each cryoprotective additive and by reducing the exposure time of embryos/oocytes to the solution to a minimum [31].

In order to further increase the cooling rate necessary for successful vitrification, the volume of the solution in which the embryos/oocytes are vitrified has been recently dramatically decreased $(0.1-2 \mu \mathrm{l})$. To achieve this, special carrier systems (open versus closed) have been developed such as open pulled straws, Flexipet-denuding pipettes, Cryotop, electron microscopy copper grids, cryoloops or the "Hemi-Straw" system [32-34].

The application of vitrification for cryopreservation of human blastocysts and oocytes has recently been greatly increased [30].

Technically vitrification is very difficult to perform, because of the very concentrated, viscous and small volume of vitrification solutions in which the embryos/oocytes must be handled for only a very limited amount of time ( $<1 \mathrm{~min})$ prior to and during vitrification. This does not apply in the case of slow freezing, when the embryos/oocytes are cooled slowly (with a special cell freezer), because slow freezing is a more flexible technique. Similarly to slow freezing, rapid thawing is required for the optimal survival of vitrified embryos/oocytes, followed by stepwise rehydration using similar techniques employed after slow cooling [30].

Oocyte cryopreservation started more than 25 years ago. However, the success rate of oocyte cryopreservation 
improved recently with the introduction of vitrification. A recent study showed that the pregnancy rate using fresh oocytes is no better than using frozen donors oocytes, with a 50\% pregnancy rate in both groups [29]. A meta-analysis concluded that the live-birth rate per oocyte was significantly lower with slow-frozen oocytes compared to vitrified oocytes $[35,36]$. The pregnancy rate from vitrified autologous oocytes was $38 \%$ compared with $60 \%$ from vitrified donor oocytes [37-40]. The odds ratio for fertilization was similar for vitrification compared with fresh oocytes and was in favor of vitrification compared with slow freezing [36].

Recently, there have been reports of successful ovarian tissue cryopreservation and transplantation. Ovarian tissue preservation will allow storage of a large number of oocytes whereas oocyte preservation will only be applicable for a limited number of oocytes. A large series of 11 fresh ovary transplants resulted in robust results (14 pregnancies and 11 healthy babies), return of menstrual cycling and normal FSH within 4-5 months after transplantation [41].

Recently, the worldwide live birth rate of $30 \%$ was recorded after transplantation for cryo-preserved ovarian tissue $[42,43]$. Women with cancers and endometriosis are at risk of POI and their conditions necessitate fertility cryopreservation options [44]. The most common benefit of ovarian transplantation is the preservation of fertility and future endocrine function in young women undergoing cancer treatment [44]. However, elective fertility cryopreservation for non-medical (social) reasons can be accepted as an option to postpone motherhood [45].

Cryo-preserved ovarian tissue can be used in healthy women who wish to preserve fertility for non-medical reasons. With vitrification methods the cryo-preserved ovarian tissue is similar to fresh ovarian tissue regarding viability and integrity $[44,45]$. Transplantation of the cryo-preserved ovarian tissue restores hormonal function and fertility [46, 47].

Gonadotropin-releasing hormone $(\mathrm{GnRH})$ treatment has been proposed to reduce the ovarian damage prior to chemotherapy (fertility chemoprotection).

A recent Cochrane review concluded that the use of GnRH agonists should be considered in women of reproductive age planned for chemotherapy [48].

\section{Future prospects}

The use of donor oocytes is the only treatment for women at risk of POI with good pregnancy outcome. Oocyte vitrification is method of fertility preservation against the ageing process. The robust results after the transplantation of the cryo-preserved ovarian tissue suggest that this method could be an alternative strategy for fertility preservation $[49,50]$.

The transplantation of cryo-preserved ovarian tissue is an outpatient procedure that restores hormonal function and fertility $[49,50]$. After ovarian tissue transplantation women are able to conceive normally without medical assistance $[51,52]$. The oocyte and ovarian tissue cryopreservation may expand the reproductive life-span for women who wish to delay childbearing.

\section{Conclusions}

The use of donor oocytes is the only treatment for women at risk of POI with good pregnancy outcome. The in-vitro-fertilization outcome improved after introduction of oocyte (or embryo) vitrification. Transplantation of the cryo-preserved ovarian tissue is an outpatient procedure, which restores hormonal function and fertility.

\section{Conflict of interest}

The authors declare no conflict of interest.

\section{References}

1. Younis JS. Ovarian aging: latest thoughts on assessment and management. Curr Opin Obstet Gynecol 2011; 23: 427-34.

2. American College of Obstetricians and Gynecologists. Committee opinion no. 502: primary ovarian insufficiency in the adolescent. Obstet Gynecol 2011; 118: 741-5.

3. Nelson LM. Clinical practice. Primary ovarian insufficiency. N Engl J Med 2009; 360: 606-14.

4. Broekmans FJ, Soules MR, Fauser BC. Ovarian aging: mechanisms and clinical consequences. Endocr Rev 2009; 30: 465-93.

5. Johnson J, Keefe DL. Ovarian aging: breaking up is hard to fix. Sci Transl Med 2013; 5: 172fs5.

6. Szafarowska M, Jerzak M. Ovarian aging and infertility. Ginekol Pol 2013; 84: 298-304.

7. Coulam CB, Stern JJ. Immunology of ovarian failure. Am J Reproduct Immunol 1991; 25: 169-74.

8. Cordts EB, Christofolini DM, Dos Santos AA, Bianco B, Barbosa $C P$. Genetic aspects of premature ovarian failure: a literature review. Arch Gynecol Obstet 2011; 283: 635-43.

9. Trans-NIH Fragile X Research Coordinating Group and Scientific Working Groups. (2008). National Institutes of Health Research Plan on Fragile X Syndrome and Associated Disorders. Rockville, MD: NIH, U.S. Department of Health and Human Services. Retrieved January 4, 2012, http://nichd.nih.gov/publications/pubs/ documents/NIH_Research_Plan_on_Fragile_X_and_Assoc_Disorders-06-2009.pdf. Last accessed 27 July 2018.

10. Rebar RW. Premature ovarian failure. Obstet Gynecol 2009; 113: 1355-63.

11. Fridovich-Keil JL, Gubbels CS, Spencer JB, et al. Ovarian function in girls and women with GALT-deficiency galactosemia. J Inherit Metabol Dis 2011; 34: 357-66.

12. Balmagambetova A, Abdelazim IA, Bekmukhambetov E, et al. Ovarian parameters and ovarian blood flow of women living in the area of environmental crisis. Eur J Obstet Gynecol Reprod Biol 2016; 200: 68-71.

13. Balmagambetova A, Zhurabekova G, Abdelazim IA, Rakhmanov S. Effect of environmental factors on ovarian reserve of women living in Aral Sea area. J Infer Reproduct Biol 2015; 3: 145-9.

14. Abdelazim IA, Abdelrazak KM, Elbiaa AA, et al. Ovarian function and ovarian blood supply following premenopausal abdominal hysterectomy. Przegl Menopauz 2015; 14: 238-42.

15. Schmidt PJ, Luff Ja, Haq NA, et al. Depression in women with spontaneous 46, XX primary ovarian insufficiency. J Clin Endocrinol Metab 2011; 96: E278-87. 
16. Kodaman PH. Early menopause: primary ovarian insufficiency and surgical menopause. Semin Reprod Med 2010; 28: 360-9.

17. Baber R, Abdalla H, Studd J. The premature menopause. In: Progress in Obstetrics and Gynaecology. Studd J (ed.), Churhill Livingstone, London 2009; 209-26.

18. De Vos M, Devroey P, Fauser BC. Primary ovarian insufficiency. Lancet 2010; 376: 911-21.

19. Shelling AN. Premature ovarian failure. Reproduction 2010; 140: 633-41.

20. Popat VB, Vanderhoof VH, Calis KA, et al. Normalization of serum luteinizing hormone levels in women with $46, \mathrm{XX}$ spontaneous primary ovarian insufficiency. Fertil Steril 2008; 89: 429-33.

21. Polyzos NP, Valachis A, Patavoukas E, et al. Publication bias in reproductive medicine: from the European Society of Human Reproduction and embryology annual meeting to publication. Hum Reprod 2011; 26: 1371-6.

22. Bidet $\mathrm{M}$, Bachelot $\mathrm{A}$, Bissauge $\mathrm{E}$, et al. Resumption of ovarian function and pregnancies in 358 patients with premature ovarian failure. J Clin Endocrinol Metab 2011; 96: 3864-72.

23. Bentzen JG, Forman JL, Larsen EC, et al. Maternal menopause as a predictor of anti-Mullerian hormone level and antral follicle count in daughters during reproductive age. Hum Reprod 2013; 28: 247-55.

24. Abdelazim IA, Belal MM, Makhlouf HH. Anti-Mullerian hormone and antral follicle count as predictors of ovarian reserve and successful IVF. Asian Pac J Reprod 2012; 2: 89-92.

25. Freeman EW, Sammel MD, Lin H, et al. Contribution of the rate of change of antimüllerian hormone in estimating time to menopause for late reproductive-age women. Fertil Steril 2012; 98: 1254-9.e1-2.

26. Nikolaou D, Lavery S, Turner C, et al. Is there a link between an extremely poor response to ovarian hyperstimulation and early ovarian failure? Hum Reprod 2002; 17: 1106-11.

27. Lawson R, El-Toukhy T, Kassab A, et al. Poor response to ovulation induction is a stronger predictor of early menopause than elevated basal FSH: a life table analysis. Hum Reprod 2003; 18: 527-33.

28. Ferraretti AP, Goossens V, Kupka M, et al. Assisted reproductive technology in Europe, 2009: results generated from European registers by ESHRE. Hum Reprod 2013; 28: 2318-31.

29. Nyboe Andersen A, Goossens V, Bhattacharya S, et al. Assisted reproductive technology and intrauterine inseminations in Europe, 2005: results generated from European registers by ESHRE: ESHRE. The European IVF Monitoring Programme (EIM), for the European Society of Human Reproduction and Embryology (ESHRE). Hum Reprod 2009; 24: 1267-87.

30. Konc J, Kanyó K, Kriston R, et al. Cryopreservation of embryos and oocytes in human assisted reproduction. Biomed Res Int 2014; 2014: 307268.

31. Rall WF, Fahy GM. Ice-free cryopreservation of mouse embryos at $-196^{\circ} \mathrm{C}$ by vitrification. Nature 1985; 313: 573-5.

32. Kasai M, Mukaida T. Cryopreservation of animal and human embryos by vitrification. Reprod Biomed Online 2004; 9: 164-70.

33. Liebermann J, Nawroth F, Isachenko Vet al. Potential importance of vitrification in reproductive medicine. Biol Reprod 2002; 67: 1671-80.

34. Vajta G, Holm P, Kuwayama M, et al. Open Pulled Straw (OPS) vitrification: a new way to reduce cryoinjuries of bovine ova and embryos. Mol Reprod Develop 1997; 51: 53-8.
35. Oktay K, Cil AP, Bang H. Efficiency of oocyte cryopreservation: a meta-analysis. Fertil Steril 2006; 86: 70-80.

36. Cobo A, Diaz C. Clinical application of oocyte vitrification: a systematic review and meta-analysis of randomized controlled trials. Fertil Steril 2011; 96: 277-85.

37. Smith GD, Serafini PC, Fioravanti J, et al. Prospective randomized comparison of human oocyte cryopreservation with slow-rate freezing or vitrification. Fertil Steril 2010; 94: 2088-95.

38. Rienzi L, Romano S, Albricci L, et al. Embryo development of fresh 'versus' vitrified metaphase II oocytes after ICSI: a prospective randomized sibling-oocyte study. Hum Reprod 2010; 25: 66-73.

39. Cobo A, Kuwayama M, Pérez $\mathrm{S}$, et al. Comparison of concomitant outcome achieved with fresh and cryopreserved donor oocytes vitrifi ed by the Cryotop method. Fertil Steril 2008; 89: 1657-64.

40. Cobo A, Meseguer M, Remohí J, Pellicer A. Use of cryo-banked oocytes in an ovum donation programme: a prospective, randomized, controlled, clinical trial. Hum Reprod 2010; 25: 2239-46.

41. Silber SJ. Ovary cryopreservation and transplantation for fertility preservation. Mol Hum Reprod 2012; 18: 59-67.

42. Andersen CY, Silber SJ, Bergholdt SH, et al. Long-term duration of function of ovarian tissue transplants: case reports. Reprod Biomed Online 2012; 25: 128-32.

43. Donnez J, Dolmans MM, Pellicer A, et al. Restoration of ovarian activity and pregnancy after transplantation of cryopreserved ovarian tissue: a review of 60 cases of reimplantation. Fertil Steril 2013; 99: 1503-13.

44. Kagawa N, Silber S, Kuwayama M. Successful vitrification of bovine and human ovarian tissue. Reprod Biomed Online 2009; 18: 568-77.

45. Garcia-Velasco JA, Domingo J, Cobo A, et al. Five years' experience using oocyte vitrification to preserve fertility for medical and nonmedical indications. Fertil Steril 2013; 99: 1994-9.

46. Silber S, Kagawa N, Kuwayama M, Gosden R. Duration of fertility after fresh and frozen ovary transplantation. Fertil Steril 2010; 94: 2191-6.

47. Lockwood GM. Social egg freezing: the prospect of reproductive 'immortality' or a dangerous delusion? Reprod Biomed Online 2011; 23: 334-40.

48. Chen H, Li J, Cui T, Hu L. Adjuvant gonadotropin-releasing hormone analogues for the prevention of chemotherapy induced premature ovarian failure in premenopausal women. Cochrane Database Syst Rev 2011; 9: CD008018.

49. Hilders CG, Baranski AG, Peters L, et al. Successful human ovarian autotransplantation to the upper arm. Cancer 2004; 101: 2771-8.

50. Kim SS, Hwang IT, Lee HC. Heterotopic autotransplantation of cryobanked human ovarian tissue as a strategy to restore ovarian function. Fertil Steril 2004; 82: 930-2.

51. Oktay K, Buyuk E, Veeck L, et al. Embryo development after heterotopic transplantation of cryopreserved ovarian tissue. Lancet 2004; 363: 837-40

52. Rosendahl M, Loft A, Byskov AG, et al. Biochemical pregnancy after fertilization of an oocyte aspirated from a heterotopic autotransplant of cryopreserved ovarian tissue: case report. Hum Reprod 2006; 21: 2006-9. 